Erschienen in:

José-Antonio Calañas Continente und Robles i Sabater, Ferran (Hg.): Die Wörterbücher des Deutschen: Entwicklungen und neue Perspektiven. Frankfurt am Main u.a: Lang (Spanische Akzente - 2), S. 325-344

Christine Möhrs

\title{
Syntagmatische Verwendungsmuster in einsprachigen deutschen Wörterbüichern. Teilergebnisse aus einer Vergleichsanalyse
}

\begin{abstract}
This paper presents some results from a study of 10 monolingual German dictionaries (including general dictionaries, dictionaries for learners of German as a foreign language, and dictionaries specialized in syntagmas) concerning the treatment of syntagmatic patterns. A brief introduction with regards to content and terminology is followed by an analysis of the syntagmatic patterns for the head word Kaffee in all dictionaries. A discussion of the results shows that the different dictionary types differ in the selection and the presentation of syntagmatic patterns.

Finally, some further results of the study will be presented and additional research ques. tions will be raised.
\end{abstract}

Zusammenfassung - In diesem Beitrag werden Teilergebnisse aus einer Untersuchung von zehn einsprachigen deutschen Wörterbüchern (darunter Allgemeine Bedeutungswörterbücher, Lernerwörterbücher und auf Syntagmen fokussierte Spezialwörterbücher) und den darin befindlichen syntagmatischen Verwendungsmustern präsentiert. Zunächst erfolgt eine inhaltliche und terminologische Hinführung. Im Anschluss werden Analyseergebnisse anhand des Beispiellexems Kaffee zusammengefasst dargestellt und diskutiert. Außerdem wird gezeigt, dass Unterschiede in der Auswahl und Aufbereitung der syntagmatischen Verwendungsmuster in Hinblick auf die verschiedenen Wörterbuchtypen bestehen.

Ein Einblick in einige zusammenfassende Ergebnisse sowie weitere Forschungsfragen runden den Beitrag ab.

\section{Einleitung}

Den theoretischen Ausgangspunkt des Beitrags' bildet die Annahme, dass es nicht ausreicht, sich schriftlich oder mündlich angemessen äußern zu können, wenn ein Sprecher oder eine Sprecherin nur einzelne Wörter einer Sprache kennt. „Nicht nur die korrekte Verwendung eines Worts, sondern schon für die Wahrnehmung seiner Bedeutung ist es unerlässlich, den Verwendungskontext dieses Wortes zu kennen" (Model 2010: 17).

1 Die Ausführungen und Teilergebnisse beruhen auf der Dissertation der Verfasserin (Titel: „Syntagmatische Verwendungsmuster in einsprachigen deutschen Wörterbüchern"), die an der Leibniz Universität Hannover entstanden und eingereicht ist. 
Bei manchen Wortverbindungen im Deutschen ist eine Zuordnung, ob es sich dabei um eine lose oder eine feste Wortverbindung handelt, eindeutig zu treffen (eine Schnitte, zwei Stück Kuchen, ein paar Süßigkeiten e[ssen] [vgl. Abb. 3] vs. essen wie ein Spatz [vgl. Abb. 1]). Bei anderen Wortverbindungen scheint die Grenze zwischen loser und fester(er) Wortverbindung fließender (vgl. z. B. heute Abend werde ich warm (warme Speisen) essen [vgl. Abb. 2] $]^{3}$ ), sodass man sich bei dem Versuch einer grundsätzlichen Klassifizierung - und damit einer Trennung bzw. Aufgliederung unterschiedlicher Wortverbindungen - auf einem schmalen Grad bewegt (vgl. Hollós 2010).

es-sen <isst, aß, hat gegessen> I. mit OBJ/ohne
OBJ घjmd. isst otwas durch Kauen und Schlucken
Nahrung aufnehmen Sie essen gern Gemüse/kein
Fleisch., Er aß genussvoll/hastig/im Stchen/unre-
gelmäßig. II. ohne OBJ jmd. isst irgendwio eine
Mahlzeit zu sich nehmen, die nach einer genannten
Zubereitungsart gekocht ist Wollen wir französisch/
italienisch/griechisch/vegetarisch/mittags warm/
abends kalt essen? wessen wie ein Spatz: sehr
wenig essen Es wird nichts so heil gegessen, wie
es gekocht wird.: Man stellt sich etwas viel
schlimmer vor, als es ist. Essen und Trinken hălt
Leib und Seele zusammen.: Man muss essen, wenn
man bei Krdften bleiben will.

Abb. 1: Wortartikel essen (PGWDaF)

2 Im Wörterbuchwortartikel zu dem Lexem essen, der in Abb. 3 abgebildet ist, wurde das Lexem innerhalb des syntagmatischen Verwendungsmusters aus Platzgründen abgekürzt. Um den Lesefluss zu erleichtern, ist in diesem Beispiel und in den folgenden Beispielen bei Abkürzung das Lexem von der Verfasserin ausgeschrieben und der ergänzte Wortteil in eckige Klammern gesetzt worden.

3 Wie in dem Wortartikel essen aus dem DUDEN-DAF in Abb. 2 zu erkennen ist, wird hier der Uneinheitlichkeit bei der Klassifizierung dieser Wortverbindung versucht dadurch Rechnung zu tragen, indem eine Erläuterung hinzugefügt wird (in runden Klammern eingefügt warme Speisen). Diese Vorgehensweise in den analysierten Wörterbüchern wird auch für das später ausgeführte Beispiel zu Syntagmen zum Lexem Kaffee noch näher beleuchtet. 
Die Wortverbindungen, die in diesem Beitrag fokussiert behandelt werden, sind im Folgenden unter dem Begriff, syntagmatisches Verwendungsmuster' gefasst. Eindeutige idiomatische Wendungen, Redewendungen oder Sprichwörter werden aus den Beobachtungen ausgeklammert. Eine Arbeitsdefinition, die im weiteren Verlauf des Beitrags dem 'Terminus, syntagmatisches Verwendungsmuster' zugrunde gelegt wird, lautet:

Unter einem syntagmatischen Verwendungsmuster wird eine Kombination von Wörtern verstanden, also sowohl zwei- als auch mehrgliedrige Wortverbindungen sowie satzförmige Muster. Es handelt sich um Wortverbindungen, die von ihrem Festigkeitsgrad her als lose eingestuft werden, wobei eine graduelle Abstufung zu festeren Wortverbindungen angenommen wird.

es|sen ['Esn], isst, aß, gegessen: a) (itr ) [feste] Nahrung zu sich nehmen: hastig essen und trinken; im Restaurant, bei Kerzenlicht essen; ich habe noch nicht zu Mittag gegessen; heute Abend werde ich warm (warme Speisen) essen; sich satt essen (essen, bis man satt ist); an einer Gans drei Tage essen. Syn.: das Essen einnehmen, dinieren, fressen (derb), futtern (ugs.), mampfen (ugs.), schlemmen, schmausen, schwelgen (geh.), sich den Bauch vollschlagen (ugs.), sich den Wanst vollschlagen (salopp abwertend), soupieren (geh.), speisen (geh.), sich stärken, tafeln (geh.), sich vollfressen (salopp). b) (tr.; hat) als Nahrung zu sich nehmen, verzehren: einen Apfel essen; sie isst kein Fleisch; was gibt es heute zu essen? Syn.: fressen (derb), futtern (ugs.), genießen (geh.), mampfen (ugs.), nehmen (geh.), sich den Bauch vollschlagen mit (ugs.), sich den Wanst vollschlagen mit (salopp abwertend), speisen (geh.).

Abb. 2: Wortartikel essen (Duden-DaF)

In einsprachigen deutschen Wörterbüchern, genauer Sprachwörterbüchern ${ }^{4}$, sind syntagmatische Verwendungsmuster aufbereitet und aufgelistet. Sie wurden als Quelle für dieses Phänomen ausgewählt. Die Fähigkeit zur Kombination sprach-

4 "Ein Sprachwörterbuch ist ein Nachschlagewerk, das vornehmlich dazu gemacht ist, daß seine Benutzer vornehmlich zu denjenigen sprachlichen Ausdrücken (=Lemmata) Informationen erhalten, unter denen sie nachschlagen. Die Lemmata sind entweder einer 
licher Elemente zu komplexen Mustern stellt eine wichtige Kompetenz sowohl bei L1 - als auch besonders bei L2-Sprechern dar. Syntagmatische Verwendungsmuster sind daher aus linguistischer Sicht von großem Interesse, da sie "wie kaum eine andere Konstruktion sowohl lexikalische als auch syntaktische Beziehungen in einem kontextuellen Ausschnitt illustrieren" (Storjohann 2011: 123). Nach Schafroth (2011) ist im Kontext vom Fremdspracherwerb die Verfügbarkeit von Lexemen nicht das Hauptproblem, „sondern die übliche Kombination mit anderen Lexemen" (Schafroth 2011: 67). Daher sollten „Wörterbücher für Lerner [...] im Idealfall eine sehr starke syntagmatische Komponente enthalten [... ${ }^{\text {a }}$ (Schafroth 2011: 68).

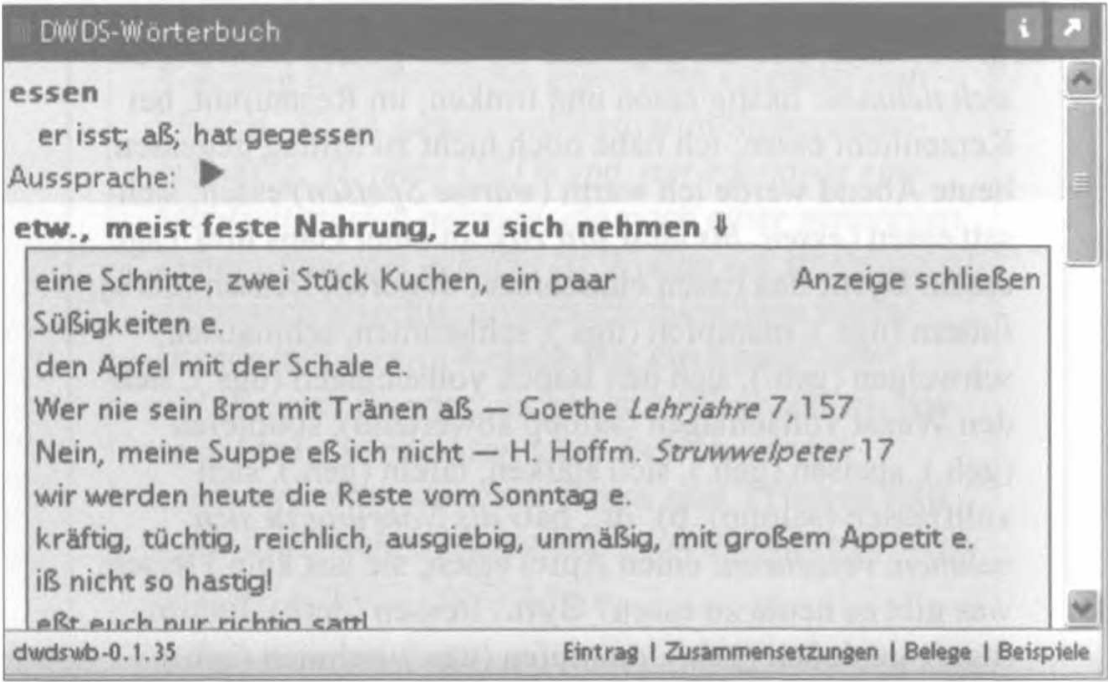

Abb. 3: Ausschnitt aus dem Wortartikel essen (dwds-WDG)

\section{Wörterbuchanalyse}

Die zentralen Forschungsfragen, die im Kern dieser Arbeit behandelt werden, lauten in Bezug auf die Untersuchung der syntagmatischen Verwendungsmuster aus den ausgewählten Wörterbüchern:

alphabetischen oder einer anderen Ordnung unterworfen und können Abkürzungen. Wortstämme, Wörter, Wortverbindungen und Wortgruppen sein“ (Wiegand 1977: 57). 
- Wie wird in einsprachigen Wörterbüchern der deutschen Sprache der syntagmatische Kontext ${ }^{5}$ eines Lexems - genauer der Kotext, also der für eine Textstelle vorausgehende bzw. folgende textinterne sprachliche Kontext - terminologisch erfasst und beschrieben?

- Welche typografischen Besonderheiten gibt es in den ausgewählten Werken?

- Welche Unterschiede bestehen für die Wortarten Nomen, Verb und Adjektiv in formaler Hinsicht in der Auswahl der Verwendungsmuster in Wörterbüchern mit andersgearteter Inhalts- bzw. Zielgruppenausrichtung?

Um sich Antworten zu den unter 1) und 2) formulierten Fragen zu nähern, sind für einen detaillierten Vergleich zehn einsprachige Wörterbücher ${ }^{6}$ zum Deutschen ausgewähit worden:

\section{Allgemeine Bedeutungswörterbücher:}

Print-Wörterbücher:

- Duden - Universalwörterbuch (= DUDEN-UW)

- Wahrig - Deutsches Wörterbuch (= WAHRIG)

Online-Wörterbücher:

- elexiko (<www.elexiko.de>) (= ELEXIKo)

- dwds-WDG (besonders die Betaversion) (<www.dwds.de $>$ ) (= DwDs-WDG)

- Wiktionary (<http://de.wiktionary.org/wiki/Main_Page >) (= WIKTIONARY)

Lerner-Wörterbücher:

- Duden - Deutsch als Fremdsprache (= Duden-DAF)

- PONS - Großwörterbuch Deutsch als Fremdsprache (= PGWDAF)

- Langenscheidt - Großwörterbuch Deutsch als Fremdsprache (= LGWDAF) Spezialwörterbücher:

- Duden - Stilwörterbuch (=Duden-STILwв)

- E. Agricola: Wörter und Wendungen (= WörTer und Wendungen)

Mit dieser Auswahl, mit der selbstverständlich kein Anspruch auf Vollständigkeit erreicht werden kann, sind neben Werken für Muttersprachler auch solche für

5 Zum Begriff Kontext stellt Franck fest: „Weil der Text etwas relativ Greifbares zu sein scheint, mag sich dies in gewissem Maß auf den Kontext übertragen, vor allem dann, wenn man Kontext primär wiederum als Text auffaßt, d. h, den schriftlichen Kontext, der den jeweils betrachteten Text(-teil) umgibt. Erst im Laufe der Entwicklung der Textlinguistik wurde für diese textuelle Umgebung der Terminus ,Kotext' geprägt, in Unterscheidung vom allgemeinen Kontextbegriff, der den außersprachlichen, situativen Kontext umfaßt" (Franck 1996: 1324).

6 Im Folgenden werden für die Wörterbücher die in Klammern hinter dem Gleichzeichen eingeführten Abkürzungen verwendet. 
Lerner abgedeckt. Es befinden sich darunter Wörterbücher, in denen ein breites Spektrum an Angaben enthalten ist, sowie solche, die eine spezielle inhaltliche Ausrichtung haben. Zudem wurden gedruckte und online publizierte Werke herangezogen.

Für die Beantwortung der unter 3) gestellten Frage wurden mittels wortartenspezifischer und wortartenübergreifender, formaler Kriterien Detailuntersuchungen von je zehn Wortartikeln der Wortarten Nomen, Verb und Adjektiv und den darin aufgeführten Syntagmen durchgeführt. Die Analyse der syntagmatischen Verwendungsmuster nach diesen Kriterien soll es ermöglichen, quantitative Aussagen über die formale Struktur und über möglicherweise vorhandene Unterschiede in Bezug auf Wörterbücher mit unterschiedlicher Inhalts- bzw. Zielgruppenausrichtung machen zu können.

Wortverbindungen spielen - ganz besonders in Wörterbüchern, die speziell für Lerner ausgerichtet sind - eine große Rolle (vgl. z. B. Storjohann 2011, Schafroth 2011). Abel (2000) begründet dies folgendermaßen:

Man scheint sich zunehmend dessen bewusst zu werden, dass Bedeutungserklärungen nicht alles erklären können und dass Beispiele einen Teil der Bedeutungserklärung übernehmen müssen. Und außerdem impliziert die Kenntnis der Bedeutung eines Wortes keineswegs das Wissen um die Verwendungsmöglichkeiten. (Abel 2000: 164)

Der Angabebereich der syntagmatischen Verwendungsmuster ist in vielen deutschen Wörterbüchern umgesetzt worden - allerdings auf unterschiedliche Art und Weise. Dieser Bereich wurde für einsprachige Wörterbücher, wie beispielsweise in Langenscheidts Grosswörterbuch Deutsch als Fremdsprache (vgl. Kühn 1998 oder Lehr 1998), im De GruYter Wörterbuch Deutsch als FREMDSPRACHE (vgl. Köster / Neubauer 2002 oder Heine 2005) oder auch für zweisprachige Wörterbücher mit Deutsch als einer der zwei Sprachen im Rahmen einer Dissertation (vgl. Model 2010) untersucht.

Sich mit syntagmatischen Verwendungsmustern, also mit diesen loseren Wortverbindungen, noch genauer auseinanderzusetzen, ist wichtig, denn diese sprachlichen Formen werden

von Lexikografen, Fremdsprachendidaktikern und Sprachtheoretikern als fundamental erachtet, da sie zwar unter semantischem Gesichtspunkt nicht idiomatisch, wegen ihrer mehr oder weniger ausgeprägten Festigkeit aber doch auffällig und problematisch sind [...]. Die Phraseologieforschung behandelt sie stiefmütterlich. Die Kollokationsforschung schenkt ihnen aufgrund ihres Mehr-als-zwei-Wort-Charakters noch nicht die nötige Aufmerksamkeit. (Hollos 2010: 86) 


\section{Wörterbuchwortartikelanalyse bei Nomen}

Für die Analyse der Wortart Nomen, die in diesem Beitrag ausschnittsweise präsentiert wird, wurden sechs Oberkriterien ausgewählt, die sich in drei Bereichen in Unterkriterien aufschlüsseln (vgl. Tab. 1):

Tab. 1: Analysekriterien der Wortart Nomen

\begin{tabular}{|c|c|}
\hline Oberkriterien & Unterkriterien \\
\hline Anzahl der Muster, & -- \\
\hline variable Elemente, & - \\
\hline Erläuterungen, & - \\
\hline Verbalphrasen, & infinite und finite Verbalphrasen \\
\hline Artikelgebrauch, & $\begin{array}{l}\text { ohne Artikel, } \\
\text { mit Artikel: } \\
\text { - mit definitem und mit indefinitem Artikel, } \\
\text { - Personal- bzw. Zahlpronomen anstelle } \\
\text { eines Artikels }\end{array}$ \\
\hline Numerus. & $\begin{array}{l}\text { Singular } \\
\text { Plural }\end{array}$ \\
\hline
\end{tabular}

Die prozentuale Verteilung bezieht sich bei den Kriterien der 1. Ebene auf die Gesamtheit aller syntagmatischen Verwendungsmuster. Die Bezugsgröße bei den Kriterien der 2. Ebene ist das jeweilige Oberkriterium.

Die Kriterien der Wortart Nomen werden nun im Einzelnen beschrieben und ihre Relevanz für die Analyse herausgearbeitet. Im Anschluss erfolgt eine Darstellung der Teilergebnisse anhand der Untersuchung der syntagmatischen Verwendungsmuster des Beispiellexems Kaffee.

\subsection{Anzahl der Muster}

Zunächst wird die Anzahl der syntagmatischen Verwendungsmuster ermittelt, die in den untersuchten Wörterbüchern zu dem jeweiligen Lexem aufgelistet sind. Diese Zahl dient bei der Analyse der Einzellexeme, aber auch bei der Gesamtanalyse der Nomen (und später auch bei der Gesamtanalyse der drei Wortarten) als zentraler Grundwert. Auf diesen Wert beziehen sich dann die Prozentzahlen zu der Verteilung der weiteren Kriterien. Neben dieser Bedeutung als statistisch wichtiger Bezugsgröße sagt die Anzahl an gezeigten syntagmatischen Verwendungsmustern auch etwas darüber aus, in welchem Umfang dem Nutzer Syntagmen zu einem Lexem in Wörterbüchern präsentiert werden, also welcher 
Platz - das gilt besonders bei gedruckten Wörterbüchern - diesem Angabebereich bei den Nomen (aber auch bei den anderen analysierten Wortarten Verben und Adjektiven) beigemessen wird.

\subsection{Variable Elemente}

Auf der 1. Ebene wird untersucht, ob es variable Elemente innerhalb der Muster gibt. Beispiele hierfür wären syntagmatische Verwendungsmuster zum Lexem Angebot: ein günstiges, vorteilhaftes, unverbindliches, billiges A. (aus DUDEN-STILWB), Angebote von [z.B. Unternehmen] einholen (aus ELEXIKo) oder ein ablehnen, annehmen, ausschlagen (aus WAHRIG) (vgl. dazu auch die Wortartikelausschnitte in Abb. 4).

1. das Anbieten, Vorschiag: ein günstiges, verlockendes, attraktives, großaügiges, vorteilhaftes. unverbindliches, billiges $A$.; das kulturelle $A$.

Verwendungen in Verbalphrasen und sfitzen Angebote von [z. B. Unternehmen] einholen

den Waren 4 〈umg. Sondrrangebot $\bullet$ ein $\sim \mathrm{b}$ lehren, annehmen, ausschlagen; machen Sie
Worturthela mssehait ans Dedes-Stilwb

Wortartikelaweladn aw deadko

Wortartitelamedradt ave Wiahrig

Abb. 4: Wortartikelausschnitte zur Illustration von variablen Elementen

Die Darstellungsweise in den Wörterbüchern ist jeweils eine andere: Das DuDEN-STILw в listet ohne typografische Kennzeichnung die Adjektivattribute im gezeigten Beispiel mit Komma getrennt hintereinander auf. Im WAHRIG wird ebenfalls die durch Komma getrennte Auflistung gewählt. Als Orientierungshilfe für den Nutzer und um dem Benutzer "das Auffinden der gesuchten Formen zu erleichtern, werden diejenigen Wörter, die Leitwörter der Redewendungen sind, fett gedruckt" (WAHrIG, "Hinweise zur Benutzung", S. 14) (vgl. hierzu in Abb. 4 den Fettdruck des Wortes ablehnen).

Im ELEXIKo-Wörterbuch ${ }^{7}$ ist die Stelle eines variablen Elementes deutlich typografisch innerhalb eines Musters hervorgehoben. Während alle fest zum Muster gehörenden Elemente in schwarzer Schrift aufgeführt sind, erscheinen variable Elemente - durch eckige Klammern und „z. B.“ eingeleitet - in grauer, kursiver Schrift. Im gezeigten Beispiel aus ELEXIxo (Angebote von [z. B. Unternehmen] einholen; vgl.

7 Vgl. hierzu auch genauer Storjohann / Möhrs (2007) bzw. Möhrs (2011). 
Abb. 4) wird die Instanz als variabel dargestellt, die Angebote (in der Bedeutung ,Vorschlag') unterbreitet. Betrachtet man die Anzahl der angegebenen variablen Elemente, fallt auf, dass in ELEXIKo - im Vergleich zu anderen Wörterbüchern in dem Angabebereich mit syntagmatischen Verwendungsmustern die Zahl der alternativen Elemente innerhalb von Mustern jeweils auf maximal zwei Elemente begrenzt ist. Die dargebotenen Variablen, mit denen die Klammer gefüllt werden kann, scheinen aufgrund der Begrenzung auf den ersten Blick nicht so vielfältig wie in den anderen analysierten Wörterbüchern. Ein Benutzer des Online-Wörterbuchs ELEXIKo kann aber in einem anderen Angabebereich (die Rubrik ist betitelt mit „Kollokationen"), der nicht auf der gleichen Bildschirmseite zu rezipieren ist, noch weitere Variablenmöglichkeiten finden. Hier sind unter der Frage „Wer macht ein Angebot?" die Kollokationen Arbeitgeber, Club und Verein aufgelistet. Diese Kollokationen wären weitere Möglichkeiten, die Stelle des Klammerelementes aus Abb. 4 auszufüllen. Um zu dieser Erkenntnis zu kommen, hat der Wörterbuchbenutzer einige Transferarbeit zu leisten: Zunächst müsste der Benutzer ausgehend von dem Syntagma auf eine Art übergeordnete Kategorie des variablen Elementes schließen (in dem Beispiel wäre das eine übergeordnete Kategorie Personengruppe/ Institution). In einem zweiten Schritt müsste der Nutzer in der Wörterbuchrubrik "Kollokation" die Frage identifizieren, unter der auf eine Personengruppe/Institution referiert wird, die Angebote macht (in dem Beispiel wäre das die oben schon genannte Frage "Wer macht ein Angebot?"). Das im syntagmatischen Verwendungsmuster genannte Element Unternehmen wird unter der gezeigten Frage in der Rubrik „Kollokation" nicht gezeigt. Hier müsste der Wörterbuchbenutzer also in einem weiteren Schritt die unter der genannten Frage aufgeführten Kollokationen als weitere Möglichkeiten neben Unternehmen identifizieren, um die Klammer aus dem syntagmatischen Verwendungsmuster zu füllen.

Variabilität innerhalb von syntagmatischen Verwendungsmustern unterstreicht den freieren Charakter dieser Wortverbindungen. Besonders bezogen auf die Einzelwortanalyse bedeutet das: Je mehr Variabilität bei Syntagmen gezeigt wird, desto mehr wird der freiere Charakter der Wortverbindungen zu einem Wort hervorgehoben.

\subsection{Erläuterungen}

Ebenfalls auf der 1. Ebene angesiedelt ist ein Kriterium, das mit „Erläuterungen“ betitelt ist. Bei den freieren syntagmatischen Verwendungsmustern gibt es teilweise Erläuterungen, die anzeigen, dass Muster in ihrer Verwendung ( $z$. B. thematisch) eingeschränkt sind oder dass sich Teile aus dem syntagmatischen Muster bereits in einem Übergang zu einer Wortverbindung mit festerem Charakter 
befinden. Beispielsweise findet man im DUDEN-UW im Wortartikel Angst hinter dem Syntagma er hat A[ngst] in Klammern und in kursiv gesetzt eine Art Paraphrasierung zu dem Syntagma in Form einer synonymen Beschreibung: (er fürchtet sich). Das im DUDEN-UW folgende Muster enthält ebenfalls eine Form der Zusatzerläuterung, und zwar in Form einer synonymischen Umschreibung eines Teils des Syntagmas: sie hat Angst (sie befürchtet), dass alles entdeckt wird. Nicht alle Wörterbücher bedienen sich so einer Form der Erläuterung, das ELExIKOWörterbuch beispielsweise verzichtet darauf vollständig.

Erläuterungen kommen auch bei idiomatischen Syntagmen, wie $j-m$ A. [ngst] (u. Bange) machen (im Wortartikel Angst aus LGWDAF), vor. Diese werden innerhalb eines Wortartikels in der Regel getrennt von den nicht-idiomatischen, also den freieren Wortverbindungen aufgeführt und z. B. im LGWDAF durch eingeleitet. Dort werden idiomatische Wortverbindungen dann auch durch eine kurze Erläuterung, meist in Form eines Satzes, verständlich gemacht. Beim oben genannten idiomatischen Syntagma $j-m$ A. [ngst] ( $u$. Bange) machen beispielsweise folgt die kurze Erläuterung bewirken, dass $j$ - $d$ (große) Angst bekommt. Diese Form der Kurzerläuterung ist für Idiome in der Regel obligatorisch, da sich ihre Bedeutung für den Benutzer - insbesondere den nicht-muttersprachlichen Benutzer - nicht durch die Summe der Bedeutung der Einzelelemente erschließt. Solche Fälle werden aus der Analyse in dieser Arbeit jedoch ausgeschlossen, da hier gerade nicht idiomatische feste Verbindungen untersucht werden.

In der Analyse zum Bereich Erläuterungen wird überblicksartig und wortbezogen gezeigt, bei wie vielen Mustern solche Kurzerläuterungen bzw. Zusätze vorkommen, die eindeutig einen Hinweis auf eine speziellere Verwendung eines gesamten Musters oder von Teilen daraus geben. Sie sind Indizien für den Übergang von freieren zu festeren syntagmatischen Wortverbindungen, ohne dass diese bereits die Ebene der Idiomatizität vollständig erreichen.

\subsection{Verbalphrasen}

Bezogen auf die Gesamtheit aller analysierten syntagmatischen Muster wird auf der 1. Ebene gezeigt, wie viele Verbalphrasen es überhaupt bei den Nomensyntagmen gibt. In dieser Arbeit wird unter einer Verbalphrase ein syntagmatisches Verwendungsmuster verstanden, bei dem eine Konstituente des Musters ein Verb in infiniter oder finiter Form und damit der Kopf der Phrase ist (Terminologie in Anlehnung an Dürscheid 2003: 29). In einem weiteren Schritt wird außerdem auf einer 2. Analyseebene die Verteilung von infiniten und finiten Verbalphrasen ermittelt.

Es ist grundsätzlich relevant, die Verteilung von infiniten und finiten Verbalphrasen zu untersuchen, weil beide Formen dem Benutzer einen unterschiedlichen 
sprachlichen Gebrauch vorführen, dem Benutzer von der Auffassungsgabe her Unterschiedliches abverlangen und sie somit auch in ihrer Funktion variieren.

Infinite Verbalphrasen aus Wortartikeln zu Glück wie sein G[lück] verscherzen (aus WöRTER UND WENDUNGEN), (kein, wenig, viel) G[lück] (in der Liebe, im Spiel) haben (aus LGWDAF) oder sich an den A[rzt] wenden (im Wortartikel Arzt aus DWDS-WDG) illustrieren in ihrer Infinitheit einen sehr abstrakten Sprachgebrauch. Der Benutzer muss beispielsweise bei dem Muster sein G[lück] verscher$z e n$ abstrahieren, dass das Pronomen in einem konkreten sprachlichen Kontext je nach Sprecherperspektive anders ausfällt. Besonders das Muster (kein, wenig, viel) G [lück] (in der Liebe, im Spiel) haben erfordert vom Wörterbuchbenutzer ein Verständnis dafür, wie die variablen Elemente in einer infiniten Form miteinander zu kombinieren sind, und dann das Anwendungsvermögen, aus der infiniten eine finite Verbalphrase für eine konkrete sprachliche Äußerung zu formulieren.

Bei finiten Verbalphrasen wie Wenn du G[lück] hast, ist vielleicht noch Kuchen übrig (im Wortartikel Glück aus LGWDAF), der A[rzt] untersuchte, behandelte, besuchte den Kranken (im Wortartikel Arzt aus WÖRTER UND WENDUNGEN) oder er trank hastig seinen K[affee] aus (im Wortartikel Kaffee aus DWDS-WDG) ist immer schon ein konkreter sprachlicher Ausdruck dargestellt. Zwar muss der Wörterbuchbenutzer hier den Transfer von infiniter zu finiter Verbform nicht leisten, der sprachliche Ausdruck ist aber dafür auch wesentlich starrer und in seiner konkreten Ausdrucksform fest.

Hier gilt es insbesondere im Vergleich der Wörterbuchtypen (z. B. Allgemeines Bedeutungswörterbuch vs. Lernerwörterbuch) herauszustellen, ob es zwischen ihnen Unterschiede bei der Darbietung von infiniten und finiten Verbalphrasen gibt.

\subsection{Artikel}

Ob und welche Tendenz es in Bezug auf den Artikelgebrauch zu beobachten gibt, wird im Bereich "Artikel" genauer untersucht. Auf der 1. Ebene wird analysiert, ob ein Artikel vor dem zu analysierenden Wort aufgeführt ist oder nicht. Beispiele für Syntagmen ohne Artikel vor dem Beispiellexem wären die Preise richten sich nach A/ngebot] und Nachfrage (im Wortartikel Angebot aus DUDEN-STILWB) oder in Angst sein (im Wortartikel Angst aus PGWDAF). Auf der 2. Ebene wird dann bei einem Artikelgebrauch noch in definiten vs. indefiniten Artikel spezifiziert; Beispiele für Syntagmen mit definitem Artikel sind das Glück auf die Probe stellen (im Wortartikel Glück aus ELEXIKo) oder den vollen B[eitrag] zahlen (im Wortartikel Beitrag aus DUdEN-UW), Beispiele für Syntagmen mit indefinitem Artikel sind eine Leiter aufstellen (im Wortartikel Leiter aus DUDEN-DAF) oder sich einer 
G[efahr] aussetzen (im Wortartikel Gefahr aus DWDS-WDG). Außerdem wird untersucht, wie sich vorangestellte Personal- bzw. Zahlpronomina, also z. B. seine Hoffnung hat sich erfüllt (im Wortartikel Hoffnung aus DUDEN-DAF) oder er hat in der Zeitschrift einige Beiträge veröffentlicht (im Wortartikel Beitrag aus WÖRTER UND WENDUNGEN) verteilen.

\subsection{Numerus}

In einem letzten, der 1. Ebene zugehörigen Analyseschritt wird der Gebrauch des Numerus analysiert. Hier wird die Frage verfolgt, ob ein Lexem in Syntagmen eher im Singular und im Plural verwendet wird.

\subsection{Wortartenübergreifende Kriterien}

Zu den wortartenübergreifenden Kriterien zählen die Anzahl der zu einem Lexem verzeichneten syntagmatischen Verwendungsmuster, variable Elemente innerhalb eines Syntagmas und zusätzliche Erläuterungen.

\section{Beispielanalyse Kaffee}

Insgesamt sind für das Lexem Kaffee 172 syntagmatische Verwendungsmuster in den untersuchten Wörterbüchern verzeichnet (vgl. Abb. 4).

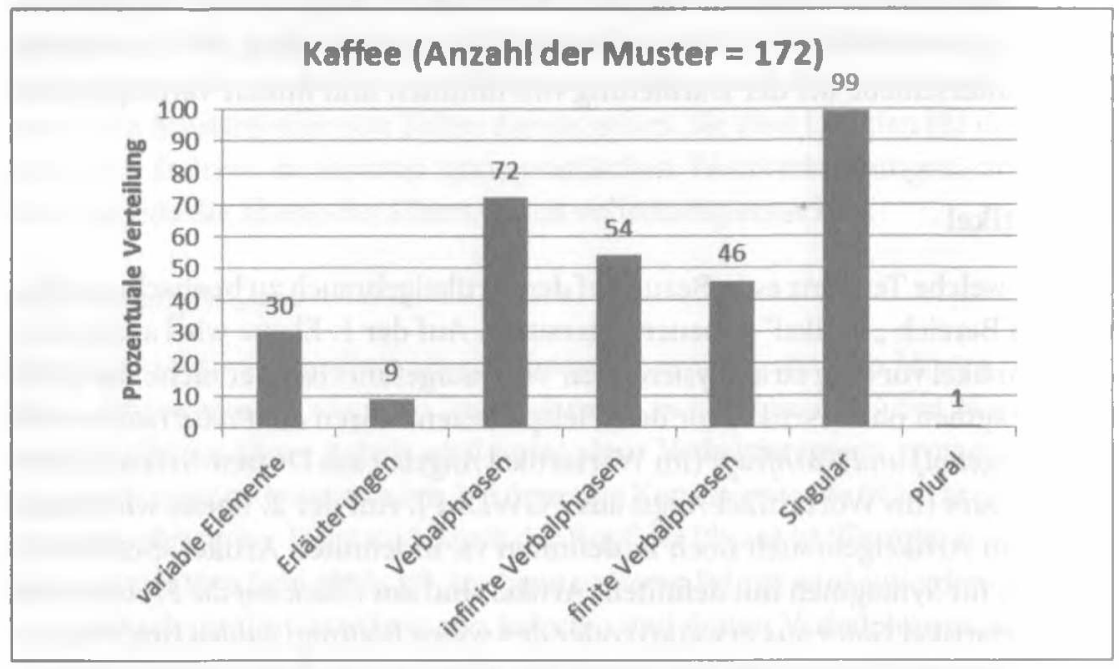

Abb. 4: Kaffee (Oberkriterien) 
Variable Elemente innerhalb der syntagmatischen Verwendungsmuster liegen in $30 \%$ der untersuchten Muster vor (vgl. Abb. 4). Im Vergleich zu den anderen Nomen, die im Rahmen der gesamten Auswertung untersucht wurden, ist Kaffee damit das Nomen mit den wenigsten Mustern, bei denen Variabilität bei Teilen aus den Syntagmen in den Wörterbüchern verzeichnet ist. Wenn Variabilität vorhanden ist, dann vor allem an der Stelle eines vorangestellten Adjektivattributes - wie in Sie hat einen heißen/schwarzen/starken/schwachen Kaffee getrunken. (aus PGWDAF) - oder beim Verb, das beschreibt, was mit dem Kaffee gemacht wird - wie in K[affee] exportieren, rösten, brennen (aus DUDEN-UW). In den häufigeren Fällen werden die Syntagmen ohne Variabilität präsentiert, wie in der Kaffee ist sehr stark (aus DUDEN-DAF) oder bitte noch eine Tasse [Kaffee]! (aus WAHRIG). Die syntagmatischen Verwendungsmuster ohne Variabilität sind in den meisten Fällen Aussagen (Beispiel aus dem DUDEN-DAF) oder wie in dem Beispiel aus dem WAHRIG Aufforderungssätze (im DUDEN-UW [Erläuterung im Beispie] unterstrichen] ist z. B. Herr Ober, zwei K[affee] (Tassen Kaffee) bitte verzeichnet). Insbesondere die Syntagmen zu Kaffee, die als Aufforderungssatz realisiert sind, haben einen starken Situationsbezug ${ }^{8}$ : Ein Sprecher oder eine Sprecherin würde solche Syntagmen in einem Café oder Restaurant der Bedienung gegenüber äuBern. Diese syntagmatischen Verwendungsmuster sind auf Grund des eindeutigen Situationsbezugs in ihrer Form festgelegter. Die geringe Variabilität innerhalb der Muster bei Kaffee unterstreicht diesen Aspekt.

Die Erläuterungen (in den nachfolgenden Beispielen unterstrichen), die zu Syntagmen von Kaffee in den untersuchten Wörterbüchern gemacht werden, beziehen sich besonders auf Syntagmen, die beschreiben, ob das Getränk Kaffee ohne oder mit irgendwelchen Zusätzen (z. B. Milch, Sahne) getrunken wird, in welchen Mengen oder auch zu welcher Gelegenheit. Hier gibt es verschiedene Gepflogenheiten, was sich eben in den Syntagmen widerspiegelt: ich mag keinen schwarzen, weißen Klaffee] (Kaffee ohne. mit Milch) (aus WÖRTER UND WENDUNGEN), ich trinke den K[affee] schwarz (= ohne Sahne) (aus DWDS-WDG); im Café ein Kännchen Kaffee bestellen (s eine Portion Kaffee. die ungefähr zwei Tassen entspricht) (aus PGWDAF); jetzt muss ich erst mal K[affee] trinken (frühstücken) (aus DUDEN-UW).

Die Realisierung der syntagmatischen Verwendungsmuster in Bezug auf die Anzahl an Syntagmen als Verbalphrase mit $72 \%$ (vgl. Abb. 5) und die Verteilung von infiniten (54\%) und finiten (46\%) Ausprägungen entsprechen in etwa der durchschnittlichen Realisierung und Verteilung bezogen auf alle analysierten

8 Dies gilt besonders für Syntagmen zu Kaffee in der Lesart, Getränk: 
Nomenmuster aus allen ausgewählten Wörterbüchern. Für das Wort Kaffee zeigt sich diese Verteilung auch beim Vergleich der einzelnen Wörterbücher (vgl. Abb. 6).

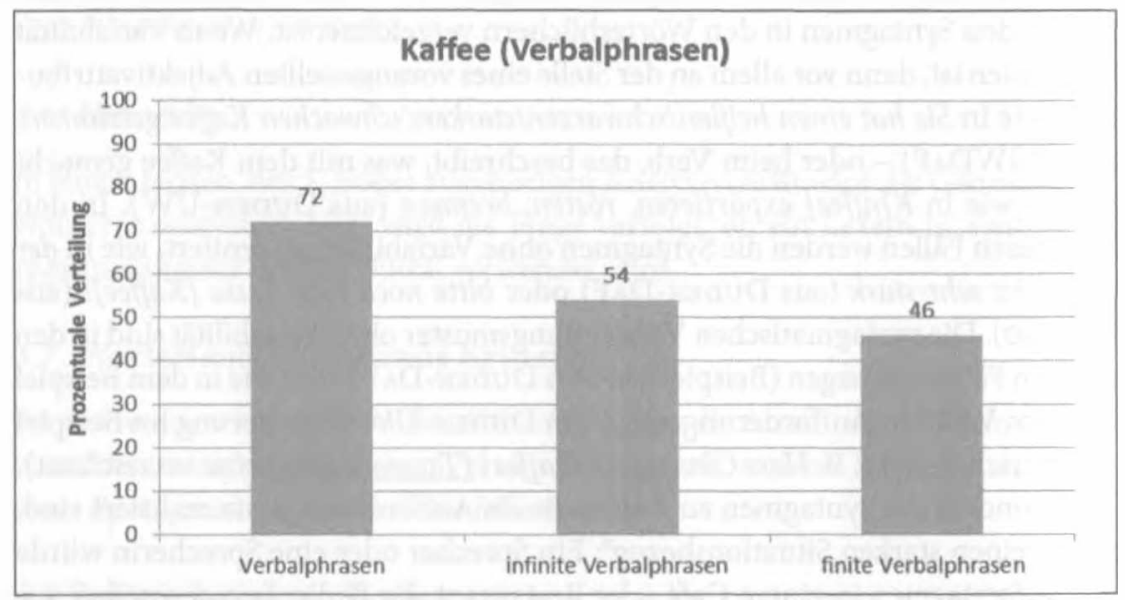

Abb. 5: Kaffee (Verbalphrasen)

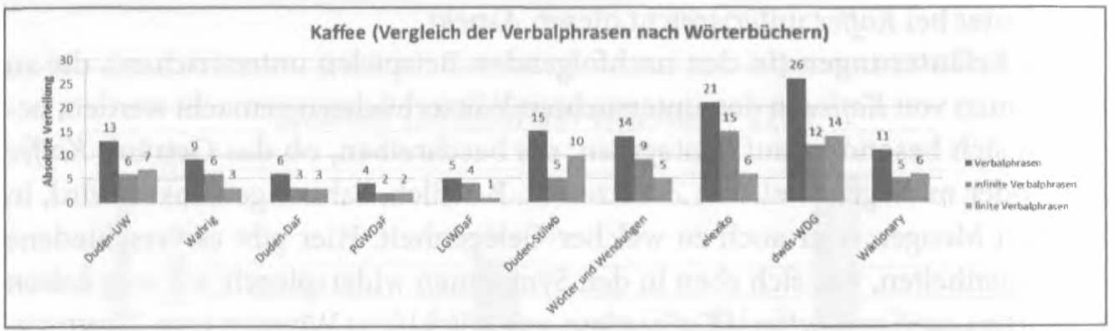

Abb. 6: Kaffee (Vergleich der Verbalphrasen nach Wörterbüchern)

Die Analyse des Artikelgebrauchs (vgl, Abb. 7) zeigt, dass in der überwiegenden Zahl der Syntagmen Kaffee ohne Artikel vorkommt (77 \%), wie z. B. in syntagmatischen Verwendungsmustern wie auf den Plantagen wird Kaffee angepflanzt (aus DUDEN-DAF) oder K[affee] ernten, exportieren, rösten, brennen, mahlen (aus Duden-STILWB). Wenn ein Artikel vorkommt, dann zumeist der unbestimmte, wie in Sie hat einen heißen/schwarzen/starken/schwachen Kaffee getrunken (aus PGWDAF). 


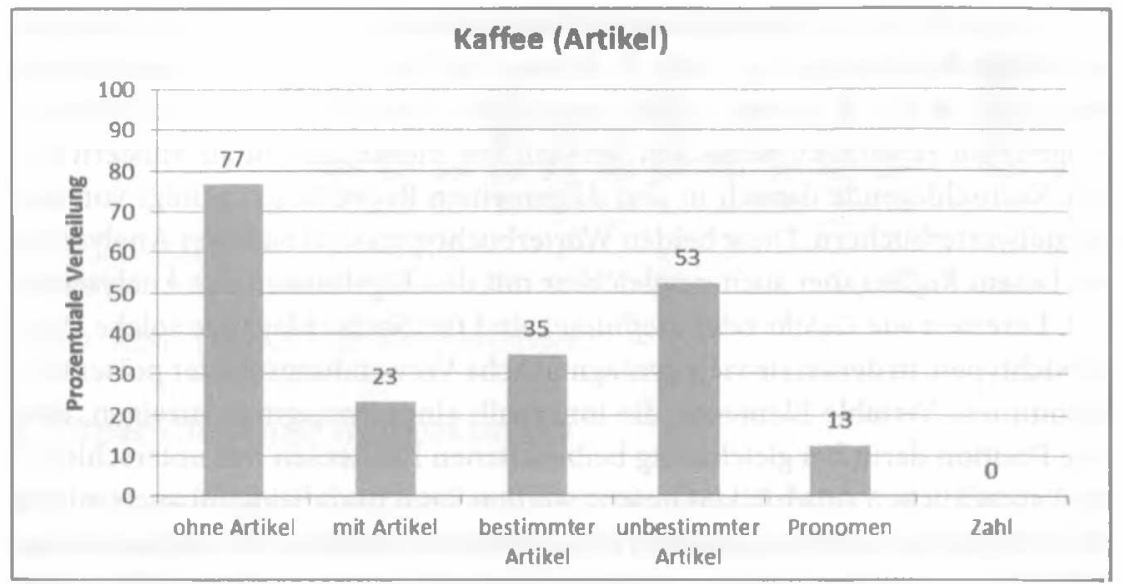

Abb. 7: Kaffee (Artikel)

Betrachtet man die Realisierung des Lexems Kaffee in Bezug auf den Numerus, fält auf, dass Kaffee in den syntagmatischen Verwendungsmustern fast ausschließlich in singularischer Form auftritt. Nur in Syntagmen, in denen Kaffeesorten thematisiert werden, kommt die Pluralform vor. Dies ist aber in den untersuchten Wörterbüchern nur ein einziges Mal der Fall: Im DUDEN-DAF (zusätzliche Erläuterung im Beispiel unterstrichen) findet man das Muster zentralamerikanische Kaffees (Kaffeesorten). Der pluralische Gebrauch des Lexems Kaffee in diesem syntagmatischen Verwendungsmuster ist als eher untypisch einzustufen. Das Aufzeigen eines solchen Musters ausschließlich in einem Lernerwörterbuch lässt vermuten, dass hier vor allem ein denkbar mögliches (aber nicht unbedingt häufiges) Syntagma mit Kaffee in pluralischer Verwendung aufgeführt ist. Die Syntagmen zeigen insgesamt den hauptsächlich singularischen Gebrauch des Lexems Kaffee.

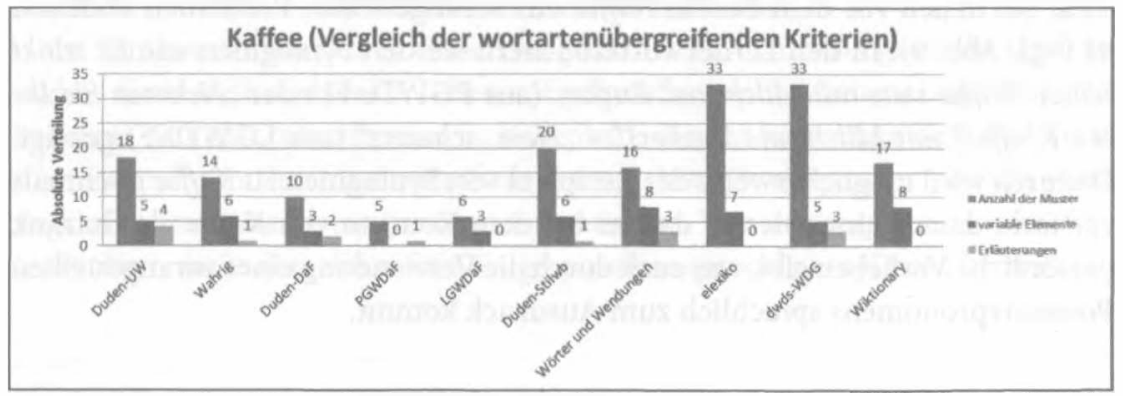

Abb. 8: Kaffee (Vergleich der wortartenübergreifenden Kriterien) 
Der Vergleich der wortartenübergreifenden Kriterien (vgl. Abb. 8) und der der Wörterbuchtypen (vgl. Abb. 9) können im Fall von Kaffee (zumindest in Bezug auf die drei Kriterien Anzahl der Muster, variable Elemente und Erläuterungen) gut zusammen betrachtet werden. Die meiste Anzahl an Mustern finden Nachschlagende danach in den Allgemeinen Bedeutungs- gefolgt von den Spezialwörterbüchern. Diese beiden Wörterbuchtypen sind nach der Analyse für das Lexem Kaffee (aber auch vergleichbar mit den Ergebnissen der Analysen zu 2. B. Lexemen wie Gefahr oder Hoffnung) sind für Nachschlagende solche Wörterbuchtypen, in denen sie viele syntagmatische Verwendungsmuster präsentiert bekommen. Variable Elemente, die innerhalb eines Syntagmas anzeigen, dass eine Position darin bei gleichzeitig beibehaltenen Elementen mit unterschiedlichen sprachlichen Ausdrücken besetzt werden kann und die damit auch immer die Komplexität der Syntagmenstruktur erhöhen, werden mit Ausnahme des PGWDAF in allen untersuchten Wörterbüchern dargeboten. Auch in Bezug auf das Kriterium der Erläuterungen zeigt sich ein relativ einheitliches Bild über die Wörterbücher hinweg betrachtet. Abgesehen von dem LGWDAF sind in fast allen Wörterbüchern Erläuterungen - zur Anzahl der Muster im jeweiligen Werk in ähnlichem Verhältnis, wenn auch grundsätzlich in recht wenigen Fällen - gegeben. Das heißt, es wird zumindest in ähnlich häufiger Weise eingestuft, dass es bestimmte syntagmatische Verwendungsmuster zu dem Lexem Kaffee gibt, die die Grenze zu einer etwas festeren Wortverbindung leicht überschreiten und damit zum Verständnis einer zusätzlichen Erläuterung bedürfen. Dass die Wörterbücher ELEXIKo und WiKTIONARY zu dem Lexem Kaffee keine Erläuterungen anbieten, ist für diese Einzelanalyse zu vernachlässigen, da darin bei allen untersuchten Nomen keine Erläuterungen angeboten werden (vgl. Abb. 8).

In Bezug auf die Wörterbuchtypen fällt außerdem besonders auf, dass in den Lernerwörterbüchern in Bezug auf das Kriterium „Artikelgebrauch“ im Vergleich zu den Allgemeinen und den Spezialwörterbüchern mehr Muster verzeichnet sind, bei denen vor dem Lexem Kaffee ein vorangestelltes Pronomen realisiert ist (vgl. Abb. 9). In den Lernerwörterbüchern werden Syntagmen wie Er trinkt seinen Kaffee stets mit Milch und Zucker. (aus PGWDAF) oder "Nehmen Sie Ihren K[affee] mit Milch und Zucker?" - „Nein, schwarz" (aus LGWDAF) gezeigt. Dadurch wird möglicherweise der Rezipient von Syntagmen zu Kaffee nochmals verstärkt darauf hingewiesen, dass es bei dem Konsum von Kaffee als Getränk persönliche Vorlieben gibt, was auch durch die Verwendung eines vorangestellten Possessivpronomens sprachlich zum Ausdruck kommt. 


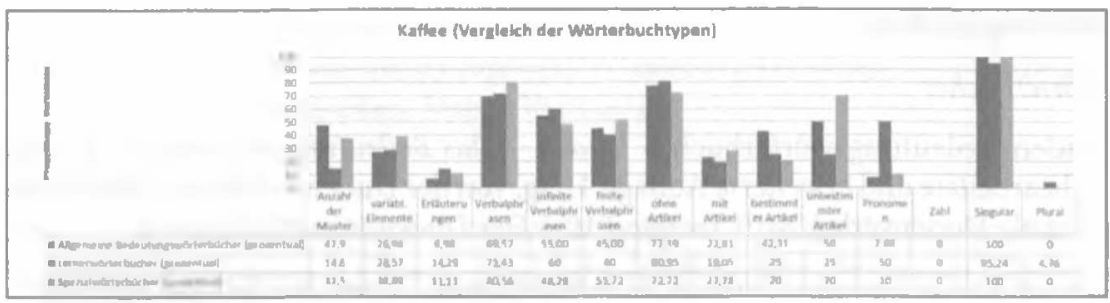

Abb. 9: Kaffee (Vergleich der Wörterbuchtypen)

\section{Abschließende Bemerkungen}

Die Untersuchung hat insgesamt zum Ergebnis, dass die Benennung, Darstellung und die in den Wörterbuchhinweisen gemachten Aussagen zur Gewinnung der in den Wörterbüchern aufgeführten Syntagmen in vielerlei Hinsicht sehr unterschiedlich ist. Besonders auffällig ist, dass in vielen der untersuchten Werke die Vorgehensweise zur Gewinnung der syntagmatischen Verwendungsmuster für den Nachschlagenden nicht aus der Rezeption der zusätzlichen Wörterbuchhinweise ersichtlich wird. Die Analyse hat bestätigt, dass zwar viele einsprachige deutsche Wörterbücher auf der Grundlage einer Datenbasis, also (großer) Textkorpora (vgl. Perkuhn / Keibel / Kupietz 2012, Bubenhofen 2006-2013), ihre Wörterbuchangaben und insbesondere syntagmatische Verwendungsmuster erarbeiten und ermitteln. Transparente Darstellungen für den Nachschlagenden in Bezug auf die Datenbasis und die Methodik zur Ermittlung der Syntagmen gibt es allerdings in den seltensten Fällen. Besonders bei dem Wörterbuch WiKTIONARY, bei dem weder die Datenbasis noch die Methode zur Ermittlung der Syntagmen für den Wörterbuchbenutzer ersichtlich wird, ist die Verlässlichkeit und die Qualität der Daten anzuzweifeln. Eine transparente Darstellung der Datenbasis und der Methoden zur Ermittlung von syntagmatischen Verwendungsmustern wäre aus Sicht der Nutzer von Wörterbüchern sehr hilfreich.?

In keinem der Werke, in denen Angaben zur Datenbasis gemacht sind, ist zudem festzustellen, dass die Daten auf gesprochen sprachlichem Datenmaterial beruhen. Für die Zukunft wäre zu bedenken, dass es besonders für Lerner und für Lehrende im Bereich Deutsch als Fremd- bzw. Zweitsprache nützlich wäre, Ergebnisse zur Auswertung von syntagmatischen Verwendungsmustern in Wörterbüchern zu finden, die auf realen gesprochen sprachlichen Daten beruhen.

9 Zur Methodik und korpusgestützten Ermittlung syntagmatischer Verwendungsmuster vgl. z. B. auch Kilgarriff u. a. 2008, Möhrs 2011). 


\section{Bibliographie}

\section{Wörterbücher}

Duden-Bedeutungswörterbuch $=$ Duden - Das Bedeutungswörterbuch. 4., neu bearbeitete und erweiterte Auflage. Hrsg. von der Dudenredaktion. Mannheim et al.: Dudenverlag 2010. (= Band 10-Der Duden in zwölf Bänden).

Duden-DaF $=$ Duden - Deutsch als Fremdsprache. 2., neu bearbeitete und erweiterte Auflage. Mannheim et al.: Dudenverlag, 2010.

Duden-Stilwb $=$ Duden - Das Stilwörterbuch. 9., völlig neu bearbeitete Auflage. Hrsg. vom Wissenschaftlichen Rat der Dudenredaktion. Mannheim et al.: Dudenverlag 2010. (= Band 2 - Der Duden in zwölf Bänden).

Duden-UW = Duden - Universalwörterbuch. 5., überarbeitete Auflage. Hrsg. vom Wissenschaftlichen Rat der Dudenredaktion. Mannheim et al.: Dudenverlag, 2003.

LGWDaF = Langenscheidt - Großwörterbuch Deutsch als Fremdsprache. Neubearbeitung. Hrsg. von Dieter Götz, Günther Haensch, Hans Wellmann. Berlin / München: Langenscheidt KG, 2010.

PGWDaF $=$ PONS - Großwörterbuch Deutsch als Fremdsprache. 1. Auflage. Bearbeitet von Dr. Andreas Cyffka et al. Stuttgart: Ernst Klett Sprachen GmbH, 2006.

Wahrig = Wahrig - Deutsches Wörterbuch. 8, vollständig neu bearbeitete und aktualisierte Auflage. Hrsg. von Renate Wahrig-Burfeind. Gütersloh / München: Wissen Media Verlag GmbH, 2006.

Wörter und Wendungen = Agricola, Erhard (Hrsg.) (1992): Wörter und Wendungen. Wörterbuch zum deutschen Sprachgebrauch. Unter Mitwirkung von Herbert Görner und Ruth Küfner u. a. Uberarbeitete Neufassung der 14. Auflage, 1. Auflage der Neufassung. Mannheim et al.: Dudenverlag.

dwds-WDG: Digitales Wörterbuch der deutschen Sprache auf der Basis des digitalisierten WDG „Wörterbuch der deutschen Gegenwartssprache ${ }^{\alpha}$ (1962-1977) im Verbund DWDS (Berlin-Brandenburgische Akademie der Wissenschaften, Berlin) <http://www.dwds.de/> sowie die dazugehörigen Projektseiten. Stand: 30.01.2015.

elexiko (2003 ff.): Online Wörterbuch zur deutschen Gegenwartssprache im Verbund OWID (Institut für Deutsche Sprache, Mannheim) <http://www. owid,de/wb/elexiko/start.html> sowie die dazugehörigen Projektseiten; Stand: 30.01.2015. 
Wiktionary DE: Wiktionary: Das freie Wörterbuch (deutsch) im Verbund Wiktionary <http://de.wiktionary.org/wiki/Wiktionary:Hauptseite> sowie die dazugehörigen Projektseiten; Stand: 30.01.2015.

Forschungsliteratur

Abel, Andrea (2000): „Das lexikographische Beispiel in der L2-Lexikographie (am Beispiel eines L2-Kontext- und Grundwortschatzwörterbuchs) ${ }^{\alpha}$. Deutsch als Fremdsprache 3, 163-169.

Bubenhofer, Noah (2006-2013): Einführung in die Korpuslinguistik: Praktische Grundlagen und Werkzeuge. Elektronische Ressource: <http://www.bubenhofer.com/korpuslinguistik/> (letzter Zugriff: 30.01.2015).

Dürscheid, Christa $\left(2003^{2}\right)$ : Syntax. Grundlagen und Theorien. Wiesbaden: VS.

Franck, Dorothea (1996): „Kontext und Kotext ${ }^{\circ}$. In: Dascal, Marcello / Gerhardus, Dietfried / Lorenz, Kuno / Meggle, Georg (Hrsg.): Sprachphilosophie. Ein internationales Handbuch zeitgenössischer Forschung. Berlin / New York: de Gruyter, 1323-1335.

Heine, Antje (2005): „Funktionsverbgefüge im Lernerwörterbuch. Ein Vergleich von ,Langenscheidt Großwörterbuch als Fremdsprache (2003) und ,De Gruyter Wörterbuch Deutsch als Fremdsprache" (2000)". In: Barz, Irmhild / Bergenholtz, Henning / Korhonen, Jarmo (Hrsg.): Schreiben, Verstehen, Übersetzen, Lernen. Zu ein- und zweisprachigen Wörterbüchern mit Deutsch. Frankfurt: Lang, 345-356.

Hollós, Zita (2010): „Auf dem schmalen Grad zwischen Kollokationen und festen Wortverbindungen“. In: I' Lexikographie. Berlin / New York: de Gruyter, 85-96.

Kilgarriff, Adam / Husák, Milos / McAdam, Katy / Rundell, Michael / Rychly, Pavel (2008): „GDEX: Automatically Finding Good Dictionary Examples in a Corpus". In: Bernal, Elisenda / DeCesaris, Janet (Hrsg.): Proceedings of the Thirteenth EURALEX International Congress. Barcelona: IULA-Universitat Pompeu Fabra, 425-432.

Köster, Lutz / Neubauer, Fritz (2002): „Kollokationen und Kompetenzbeispiele im de Gruyter Wörterbuch Deutsch als Fremdsprache". In: Wiegand, Herbert Ernst (Hrsg.), 283-310.

Kühn, Peter (1998): „LANGenscheidts Grosswörterbuch Deutsch als FremdsPRACHE und die deutschen Wörterbücher" ${ }^{4}$. In: Wiegand, Herbert Ernst (Hrsg.), 35-60. 
Lehr, Andrea (1998): Kollokationen in LANGENSCHEIDTS GROsSWÖRTERBUCH Deutsch als Fremdsprache. In: Wiegand, Herbert Ernst (Hrsg.), 254-281.

Model, Benedikt A. (2010): Syntagmatik im zweisprachigen Wörterbuch. Berlin / New York: de Gruyter.

Möhrs, Christine (2011): "Die typischen Verwendungen in elexiko". In: Klosa, Annette (Hrsg.): elexiko. Erfahrungsberichte aus der lexikographischen Praxis eines Internetwörterbuchs. Tübingen: Narr, 81-98.

Perkuhn, Rainer / Keibel, Holger / Kupietz, Marc (2012): Korpuslinguistik. Paderborn : Fink.

Schafroth, Elmar (2011): „Syntagmatische Kontexte in pädagogischen Wörterbüchern des Deutschen und Italienischen“. In: Bosco, Sandra / Costa, Marcella / Eichinger, Ludwig M. (Hrsg.): Deutsch-Italienisch: Sprachvergleiche. Heidelberg: Winter, 67-91.

Storjohann, Petra (2011): „Komplexere Gebrauchsmuster in Lernerwörterbüchern - Kritik und Perspektiven". In: Katelhön, Peggy / Settinieri, Julia (Hrsg.): Wortschatz, Wörterbücher und L2-Erwerb. Wien: Praesens, 123-144.

Storjohann, Petra / Möhrs, Christine (2007): „Das elexiko-Wörterbuch im Wandel - Typische Verwendungen neu beleuchtet ${ }^{*}$. Sprachreport 3/2007, 12-16.

Wiegand, Herbert Ernst (1977): „Nachdenken über Wörterbücher: Aktuelle Probleme". In: Drosdowski, Günther (Hrsg.): Nachdenken über Wörterbücher, Mannheim / Wien / Zürich: Bibliographisches Institut, 51-102.

Wiegand, Herbert Ernst (Hrsg.) (1998): Perspektiven der pädagogischen Lexikographie des Deutschen I. Untersuchungen anhand von "Langenscheidts Großwörterbuch Deutsch als Fremdsprache". Tübingen; Niemeyer.

Wiegand, Herbert Ernst (Hrsg.) (2002): Perspektiven der pädagogischen Lexikographie des Deutschen II. Untersuchungen anhand des "de Gruyter Wörterbuchs Deutsch als Fremdsprache". Tübingen: Niemeyer. 